\title{
Harmonic Scalpel Compared to Conventional Hemostasis in Thyroid Surgery: A Meta-Analysis of Randomized Clinical Trials
}

\author{
Adrienne L. Melck and Sam M. Wiseman \\ Department of Surgery, Saint Paul's Hospital, University of British Columbia, C303-1081 Burrard Street, \\ Vancouver, BC, Canada V6Z 1 Y6 \\ Correspondence should be addressed to Adrienne L. Melck, melckal@upmc.edu
}

Received 23 August 2009; Revised 9 November 2009; Accepted 6 December 2009

Academic Editor: Perry Shen

Copyright ( 2010 A. L. Melck and S. M. Wiseman. This is an open access article distributed under the Creative Commons Attribution License, which permits unrestricted use, distribution, and reproduction in any medium, provided the original work is properly cited.

\begin{abstract}
Background. The study's aim was to determine whether conventional hemostasis $(\mathrm{CH})$ or the Harmonic Scalpel (HS) results in shorter operative times for thyroidectomy and to evaluate the incidence of postoperative complications with each approach. Methods. A literature search was conducted from study inception to September 30, 2008. Included studies randomized thyroidectomy patients to either $\mathrm{CH}$ or $\mathrm{HS}$ and reported the incidence of postoperative transient recurrent laryngeal nerve dysfunction (RLND) and hypocalcemia. Results. Nine RCTs were included. Use of the HS reduced operative time by 23.1 minutes $(95 \% \mathrm{CI}=13.8,32.33)$. There was no difference in the incidence of transient RLND $(\mathrm{RR}=1.25,95 \% \mathrm{CI}=.56,2.76)$, but a lower rate of transient hypocalcemia with the use of the $\mathrm{HS}(\mathrm{RR}=.69,95 \% \mathrm{CI}=.51, .92)$. Conclusions. The use of HS in thyroidectomy significantly reduces operative time and is associated with a reduction in postoperative hypocalcemia compared to $\mathrm{CH}$.
\end{abstract}

\section{Introduction}

There have been few developments in the technical aspects of thyroid surgery since the surgical approach described by Kocher greater than a century ago [1]. Given the significant vascularity of the thyroid gland [2] and the relatively small operative field, meticulous hemostasis has and will always be an important prerequisite for a successful outcome in thyroid surgery. The mainstay for achieving hemostasis in thyroid surgery has been tying and/or clipping of blood vessels, both effective but time-consuming techniques. In the current climate of healthcare constraints and long surgical waiting lists, any methodology that can reduce operative times while maintaining acceptable complication rates warrants investigation.

The Harmonic Scalpel (Ethicon Endosurgery, Cincinnati, Ohio) was introduced into the surgeon's armamentarium almost two decades ago. Using mechanical vibrations at $55.5 \mathrm{kHz}$, this device is able to cut and coagulate tissue simultaneously. The proposed advantages of using this device over traditional electrocautery include less lateral thermal tissue injury, a lack of neuromuscular stimulation, and the avoidance of electrical energy transmission either to or through the patient [3]. Since the adoption of the Harmonic Scalpel (HS) into modern surgical practice, its utility for a wide variety of operations has been well documented. For example, a randomized prospective clinical trial demonstrated its ability to diminish blood loss as well as operative time for laparoscopic Nissen fundoplication [4].

Over the last decade, many reports have evaluated the utility of the HS for thyroid surgery and the majority of these studies have been carried out at European centers. The investigators have shown similar results regarding reduced operative times with its utilization, but conflicting results regarding other postoperative outcomes such as transient postoperative hypocalcemia and recurrent laryngeal nerve dysfunction (RLND). These complications are relatively uncommon and the number of cases reported in individual studies is limited. Consolidating the data may allow for elucidation of significant associations between HS utilization and postoperative complications. To date, no meta-analysis evaluating the utilization of HS in thyroid surgery has 
been reported. The purpose of this study was to determine whether conventional hemostasis $(\mathrm{CH})$ or the HS results in shorter operative times for thyroidectomy and to evaluate the incidence of postoperative complications with each approach.

\section{Methods}

2.1. Identification of Trials. We sought to identify prospective, randomized clinical trials comparing $\mathrm{HS}$ to $\mathrm{CH}$ methods (i.e., ties, clips, and/or electrocautery) for thyroidectomy utilizing a computerized literature search. We searched the Cochrane Central Register of Controlled Trials, MEDLINE and EMBASE (January 1, 1995 to September 30, 2008), using the following index terms: thyroidectomy, thyroid surgery, harmonic scalpel, harmonic shears, ultrasonic shears, ultrasonic scalpel, ultrasonic coagulator, ultrasonic dissector, ultrasonic dissection, ultrasonically activated scalpel, ultrasonic scissors, and coagulating shears. In addition, we reviewed the reference lists of retrieved articles, contacted experts in the field, and contacted the major manufacturer of the HS (Ethicon Endosurgery) to determine if they were funding or aware of any trials being conducted using their product. We also searched the proceedings of major endocrine surgery conferences for any reported trials that may not have been published. All studies were considered relevant irrespective of publication status or the language of publication.

2.2. Study Eligibility. We restricted our study to adults older than 18 years of age. Only studies comparing traditional open thyroidectomy utilizing $\mathrm{CH}$ techniques to thyroidectomy using the HS were considered. Any studies evaluating videoassisted or endoscopic thyroidectomy were excluded. Studies where additional procedures were carried out at the time of thyroidectomy (e.g., lateral neck lymph node dissection) were also excluded, unless these additional procedures were accounted for by subtracting the time for the added procedure from the overall operative time. Thyroid surgery for either benign or malignant histology was included.

The principal outcome evaluated was the mean operative time, measured in minutes, for total or subtotal thyroidectomies carried out utilizing the two surgical techniques. Although studies could include a combination of total and subtotal thyroidectomies and thyroid lobectomies, they were excluded if they did not report a mean operative time specifically for the total and subtotal thyroidectomies. The secondary outcomes we evaluated were the incidence of transient postoperative RLND and hypocalcemia. Transient RLND was not well defined in most studies. One study defined RLND as transient if vocal cord function recovered within twelve months of the operation [5]. Some studies did not provide an explanation for how transient RLND was diagnosed [6-8]. In the majority of the papers, postoperative laryngoscopy was performed on every patient to assess vocal cord function [5, 9-13]. Transient postoperative hypocalcemia was defined either by biochemical parameters or by clinical symptoms or both. Some studies did not describe how postoperative hypocalcemia was defined. Although studies could report on a variety of secondary postoperative outcomes (e.g., amount and/or duration of wound drainage, postoperative hematoma formation, pain, analgesic requirements, time to hospital discharge, costeffectiveness), they were excluded if they did not report these two specific outcomes.

To be included, studies had to be prospective, randomized clinical trials and observational studies were not included in the analysis. Clearly blinding is not feasible in studies evaluating two different surgical techniques, though it was noted if assessors of the postoperative outcomes were blinded to the intervention.

Regarding data collection and analysis, the two authors (AM and SMW) independently assessed the titles and abstracts of studies retrieved from the literature search and obtained full articles for all those that appeared to satisfy inclusion criteria, ultimately including those that met inclusion criteria after in depth review. The data from those studies were extracted independently by the authors, and any differences were resolved by discussion. The following information was abstracted for each study: year of publication, language of publication, country of origin, study design (including details on randomization, blinding, allocation concealment, intention-to-treat analysis, and losses to follow-up), provision of industrial support for the study, reason for ineligibility if the study was ultimately excluded, number of patients enrolled in each study arm, indication for thyroidectomy, type of thyroidectomy carried out (e.g., partial versus total versus subtotal), details regarding type of $\mathrm{HS}$ and $\mathrm{CH}$ utilized (ties versus clips versus electrocautery), mean operative time for total and subtotal thyroidectomies in each group, number of cases of transient and permanent postoperative hypocalcemia (either symptomatic or biochemical), number of cases of postoperative transient or permanent RLND, and number of cases of postoperative hematoma formation. Study validity is presented qualitatively though no formal validity score was assigned.

2.3. Statistical Analysis. For the primary outcome, the metaanalysis evaluated the weighted mean difference in operative times between thyroidectomy groups ( $\mathrm{HS}$ versus $\mathrm{CH}$ ) and the standard deviation of the difference from individual studies using the METAN command in STATA 9.2 (StataCorp, College Station, Texas). In one study, the data regarding operative times was not reported as a mean with standard deviation, but after correspondence with the authors, the data was provided in such a format as to allow inclusion in the analysis [11]. In 2 cases, attempts to contact the authors were unsuccessful and thus these papers could not be included in the analysis, though they had otherwise met inclusion criteria $[14,15]$. Significant heterogeneity across studies was noted; thus a pooled estimate of the difference in operative time was generated using a random effects model [16]. A sensitivity analysis excluding the two studies that disclosed financial support from the HS manufacturers was also carried out. 


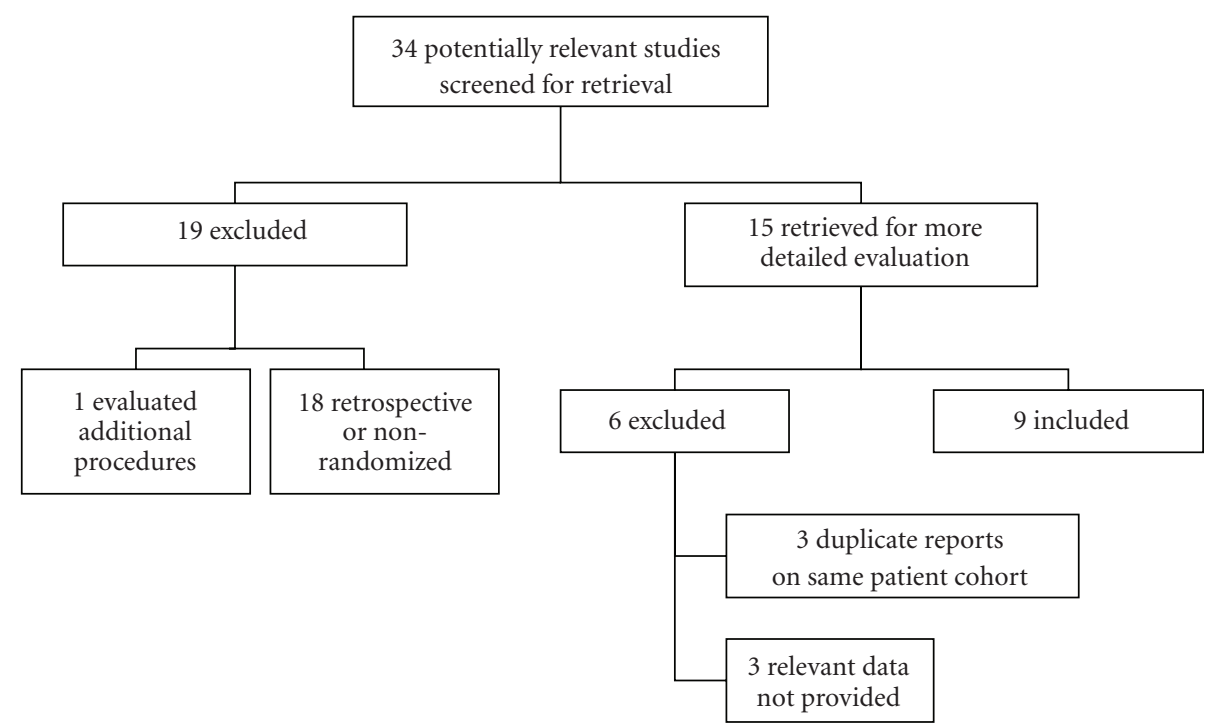

FIGURE 1: Flow diagram showing the number of studies initially identified and the reasons for study exclusion.

For the secondary outcomes of postoperative RLND and hypocalcemia, results are presented as risk ratios (RRs). The fixed effects model was utilized to obtain the summary estimates of the $\log R \mathrm{R}$ from the group of studies. We did not proceed to a random effects model once the fixed effects analysis did not reveal any significant heterogeneity (Q statistic).

Publication bias was assessed with Begg's and Egger tests and Begg's funnel plot $[17,18]$. A $P$-value of $<.05$ was considered statistically significant.

\section{Results}

Thirty-four studies that potentially met inclusion criteria were identified from the literature search. After abstract screening, 19 were excluded for variety of reasons. Of the 15 that were reviewed in depth, 6 were excluded, leaving 9 studies that were incorporated into the meta-analysis. Figure 1 depicts a flow diagram of the study selection process and Table 1 summarizes the characteristics of the studies included in the meta-analysis. There were no incidents of author disagreement in either the study selection or data extraction phase

Regarding the primary outcome of mean operative time, the pooled estimate of the weighted mean difference (WMD) in operative time obtained from a random effects model was 23.1 minutes $(95 \% \mathrm{CI}=13.8,32.33)$. This was statistically significant, with a $P$-value of $<.001$ (Figure 2 ). The $\chi^{2}$ test for heterogeneity was significant with a $P$-value of $<.001$. Tests for publication bias were not statistically significant $(P=.97)$. See Figure 3 for Begg's funnel plot.

Regarding secondary outcomes, the pooled estimate and 95\% confidence interval of the relative risk of postoperative transient RLND from a fixed effects model was $1.25(P=$ $.59 ; 95 \% \mathrm{CI}=0.56,2.76)$. Thus, there is a trend toward an increased risk of transient RLND with the use of HS, but the overall number of cases of this was small and this was not a statistically significant finding (see Figure 4). Two studies reported no cases of transient RLND [10. 12] and were excluded from this analysis. The $\chi^{2}$ test for heterogeneity was not significant $(P$-value $=.51)$; thus we did not proceed to a random effects analysis.

The pooled estimate and 95\% confidence interval of the relative risk of postoperative transient hypocalcemia from a fixed effects model was $0.69(P=.01 ; 95 \% \mathrm{CI}=0.51,0.92)$. Thus, there was a statistically significant reduced risk of transient postoperative hypocalcemia with the use of HS (see Figure 5). The $\chi^{2}$ test for heterogeneity was not significant $(P$-value $=.53)$; so a random effects analysis was not carried out.

A sensitivity analysis excluding studies with industry support revealed an even greater reduction in operative time with use of the HS ( 25 minutes; 95\% CI $=16.3,33.62$ ). Interestingly, there were a total of 3 cases of postoperative hematoma in the $\mathrm{CH}$ group and 1 in the HS group (Table 1) suggesting a trend toward a lower incidence of this serious postoperative complication with the HS. However, the overall numbers are too small to draw any meaningful conclusions.

The quality of the studies was assessed based on the following criteria: appropriateness of randomization, allocation concealment, blinding of patients, blinding of outcome assessors, utilization of intention-to-treat analysis, and a description of any patients that were lost to follow-up. In most cases, these parameters were not specified and thus the methodological quality of the included studies could only be deemed as fair. These results are presented in Table 2.

\section{Discussion}

Utilization of the HS for total and subtotal thyroidectomy significantly reduced operative time compared to $\mathrm{CH}$ 


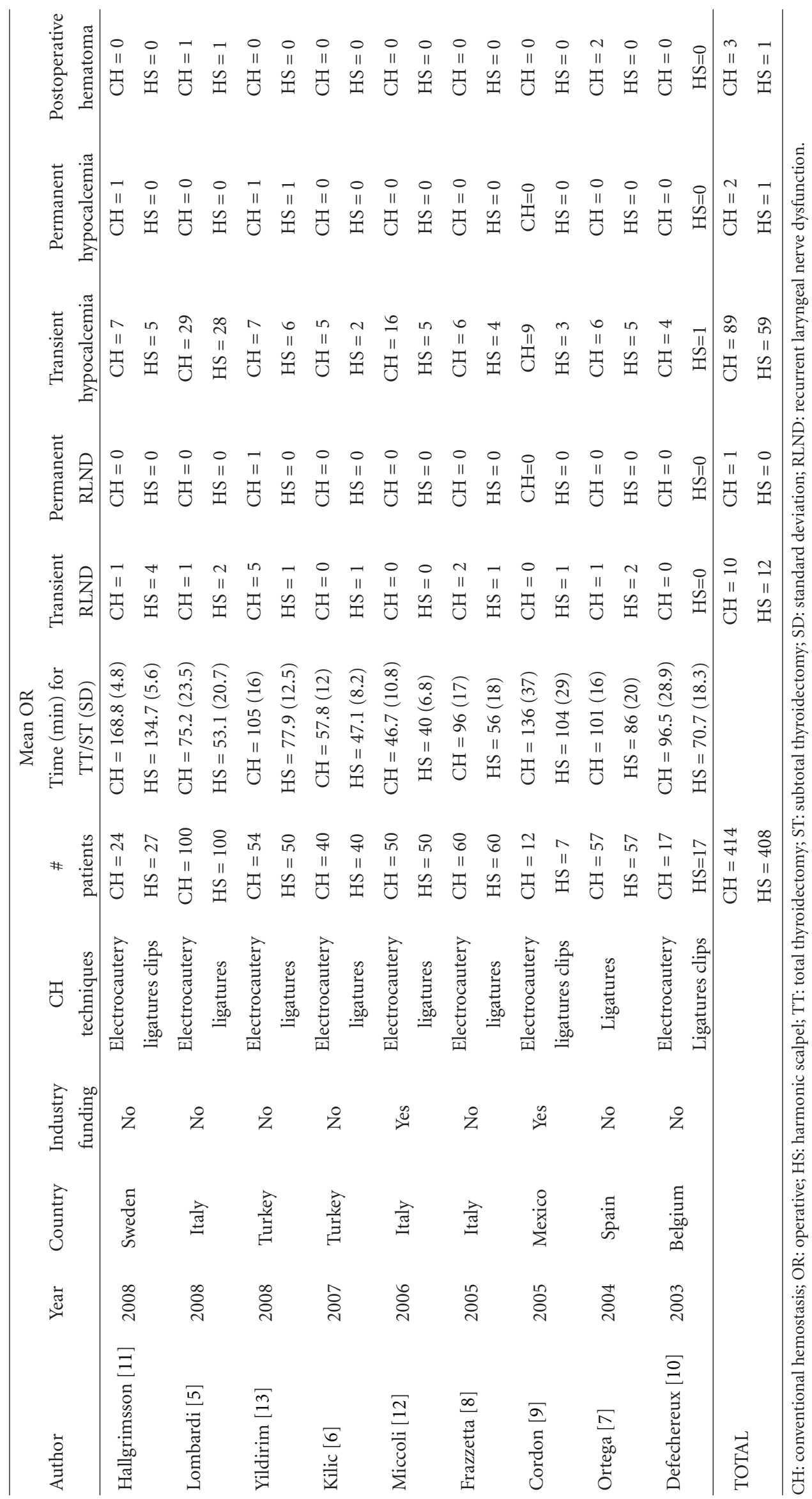




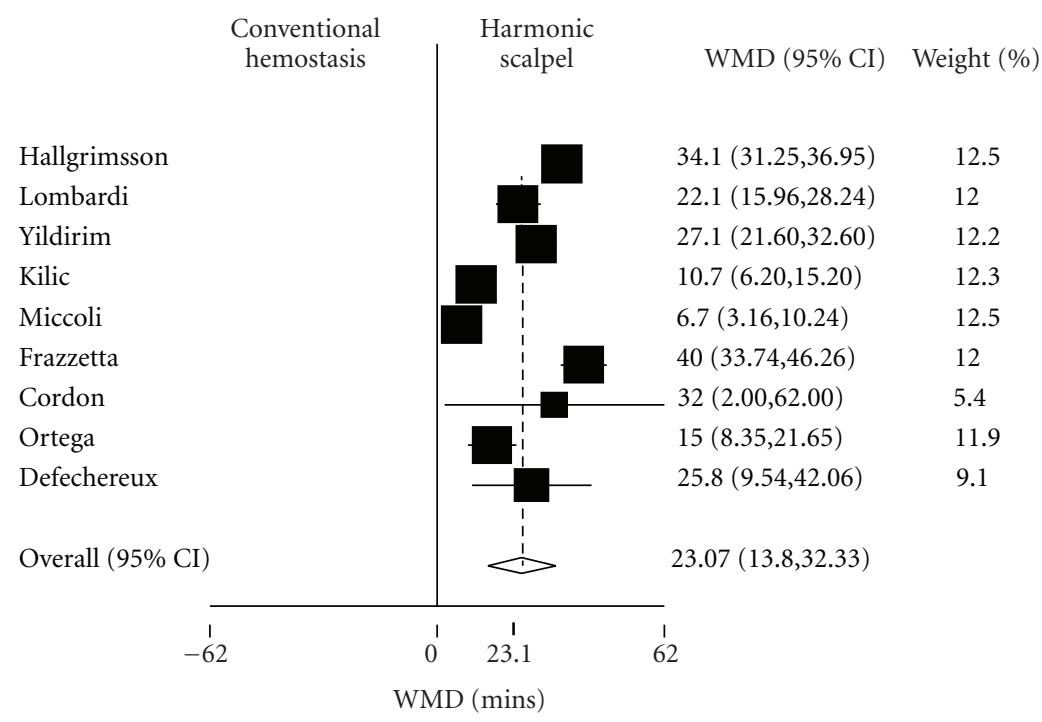

FIGURE 2: Forest plot depicting individual and pooled weighted mean difference (WMD) in operative times with $95 \%$ confidence intervals.

TABLE 2: Study validity.

\begin{tabular}{|c|c|c|c|c|c|c|c|}
\hline Author & & $\begin{array}{l}\text { zation } \\
\text { equate }\end{array}$ & $\begin{array}{l}\text { Concealed } \\
\text { allocation }\end{array}$ & $\begin{array}{l}\text { Patients } \\
\text { blinded }\end{array}$ & $\begin{array}{c}\text { Outcome assessors } \\
\text { blinded }\end{array}$ & $\begin{array}{c}\text { Intention- to-treat } \\
\text { Analysis }\end{array}$ & Patients LTFU? \\
\hline Hallgrimsson & Yes & UC & UC & UC & UC & Yes & UC \\
\hline Lombardi & Yes & UC & UC & Yes & UC & UC & UC \\
\hline Yildirim & Yes & UC & UC & UC & UC & UC & UC \\
\hline Kilic & Yes & UC & UC & UC & UC & UC & UC \\
\hline Miccoli & Yes & UC & UC & Yes & UC & UC & UC \\
\hline Frazzetta & Yes & UC & UC & UC & UC & UC & UC \\
\hline Cordon & Yes & Yes & UC & UC & Yes & UC & UC \\
\hline Ortega & Yes & UC & UC & UC & UC & UC & UC \\
\hline Defechereux & Yes & Yes & UC & UC & UC & UC & UC \\
\hline
\end{tabular}

UC: unclear; LTFU: loss to follow-up.

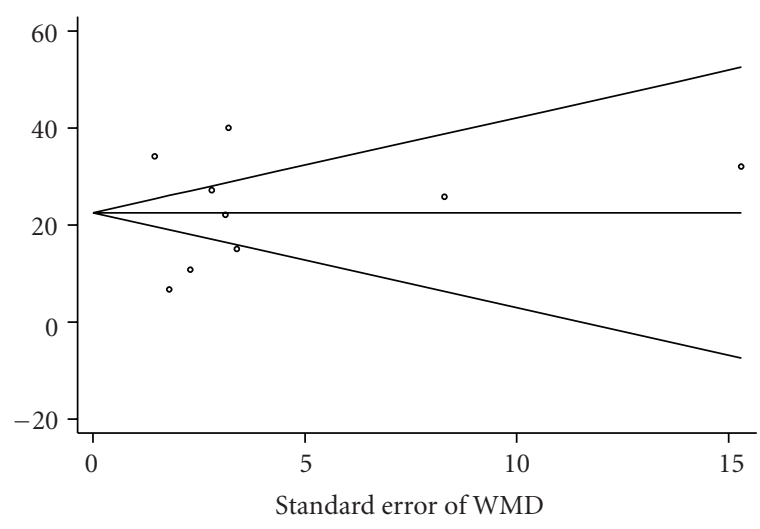

Figure 3: Begg's funnel plot with pseudo 95\% confidence limits.

techniques by greater than 23 minutes $(P$-value $<.001)$. Furthermore, there was a $31 \%$ decreased risk of transient postoperative hypocalcemia with HS utilization compared to $\mathrm{CH}$ techniques (pooled $\mathrm{RR}=0.69, P$-value $=.01$ ) and there was also no statistically significant difference in the risk of transient postoperative RLND between the two groups (pooled RR $=1.25, P$-value $=.59)$. We conclude that not only is HS utilization for total thyroidectomy significantly faster than the conventional approach, with acceptable postoperative complication rates, but also it may even protect against the development of transient postoperative hypocalcemia.

All of the studies uniformly report decreased operating time with the use of an HS. This is not a surprising observation, given that the same outcome has been reported repeatedly for a variety of other surgical procedures [19-21]. With the exception of a single Mexican study, all of the reports were from European centers. There is no reason to believe that the patients requiring thyroid surgery are any different in Europe than in North America and thus we believe that our results are generalizable to other patient populations. From the literature search, two reports from U.S. centers evaluating HS use for thyroidectomy were identified but excluded because of their retrospective study design. Both of these studies also found the HS to be safe and time-saving [22, 23]. 


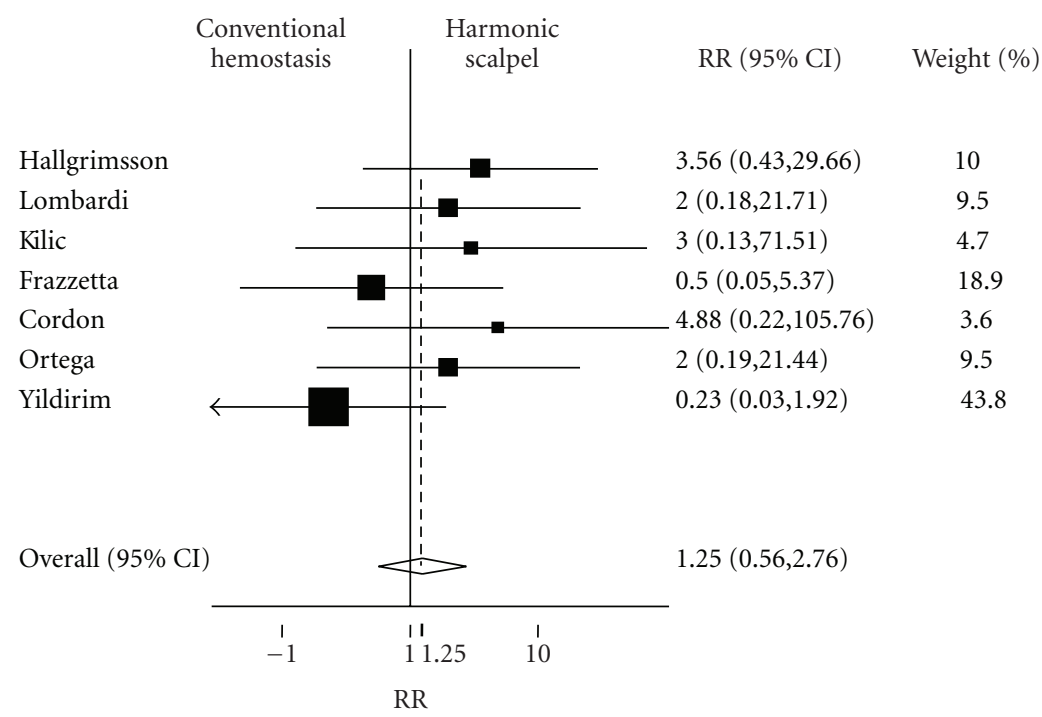

FIGURE 4: Forest plot depicting individual and pooled risk ratios (RRs) with $95 \%$ confidence intervals (CIs) for transient postoperative recurrent laryngeal nerve dysfunction.

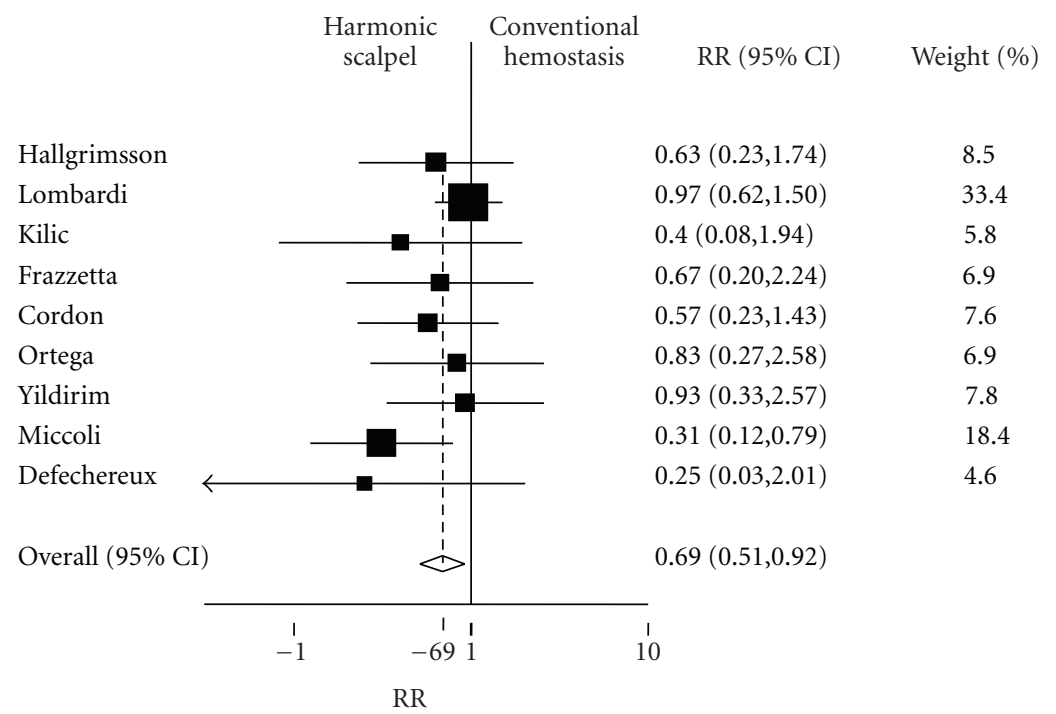

FIGURE 5: Forest plot depicting individual and pooled risk ratios (RRs) with $95 \%$ confidence intervals (CIs) for transient postoperative hypocalcemia.

All studies reported an increased risk of postoperative hypocalcemia with conventional hemostasis techniques, though only one report had a large enough cohort for the association to be statistically significant [12]. Though the mechanism is not fully understood, transient hypocalcemia observed after total thyroidectomy is believed to be related to traumatization of the parathyroid glands, which are anatomically intimately related to the thyroid gland and share its blood supply. We speculate that use of the HS may facilitate dissection of the parathyroid glands in a plane farther away from the parathyroid gland capsule, thus reducing the chance of damaging their blood supply, directly or indirectly, with either mechanical forces or electrical currents. Thus, this finding of reduced transient postoperative hypocalcemia with HS utilization does seem biologically plausible and highlights an important rationale for conducting the meta-analysis. When an outcome is relatively uncommon, individual studies may all trend toward that same outcome though none may have the power to support statistical significance, but calculating a pooled estimate may allow for the determination of a statistically significant association. It is difficult to draw any conclusions regarding permanent hypoparathyroidism and HS utilization. Permanent hypoparathyroidism is a rare 
complication of thyroidectomy, and there were only three reported cases of this among the nine studies, two of which occurred in the $\mathrm{CH}$ group and one in the HS group (Table 1).

The complication of RLND after thyroidectomy is also an extremely uncommon occurrence. Included studies had conflicting results in terms of the risks of RLND with HS utilization compared to $\mathrm{CH}$, and all reported either very few or no cases of this complication. In the current metaanalysis, there were only twenty-two incidents of transient RLND out of 822 total thyroidectomies $(.03 \%)$ or 1,644 nerves at risk $(.01 \%)$. Given that HS has been shown to cause less collateral thermal injury than conventional electrocautery, we would expect to see less RLND in the HS group. Unfortunately, the numbers in this analysis are too small to generate any meaningful conclusions. Only one case of permanent RLND occurred in a patient who underwent the $\mathrm{CH}$ technique. The time cutoff to differentiate between transient and permanent RLND was not well defined in the studies, but most investigators did use postoperative laryngoscopy in all patients to document vocal cord paralysis.

Regarding the internal validity of included studies, one must accept that for studies evaluating surgical techniques, blinding of the surgeon is not possible. However, patients can be blinded to the procedure they have undergone to minimize reporting bias when evaluating postoperative outcomes such as symptomatic hypocalcemia or pain. Furthermore, those individuals evaluating outcomes (operative time, RLND, hypocalcemia) can also be blinded to the intervention to reduce observation bias, and this was only explicitly carried out in a single study [9]. Ideally, authors should also give a detailed description of their randomization procedures, allocation concealment, and use of intentionto-treat analysis, which was not consistently reported in the studies included in this meta-analysis. To assure internal validity, future randomized studies evaluating this question should include details addressing these issues. The quality of a meta-analysis is only as good as the reports from which it is derived, and so our study is inherently limited by the methodological limitations of the included reports.

No tests of publication bias were statistically significant. Begg's funnel plot for the pooled estimate of the WMD in operative time did exhibit some asymmetry, but this was not statistically significant. The asymmetry was likely a result of between-study heterogeneity (tau-squared $=175.88$ ). When between-study heterogeneity is large and when the number of included studies is small, none of these tests to detect publication bias work well. Though all studies found that thyroidectomy was faster with the HS, they were quite heterogeneous in terms of the baseline length of time required to carry out a conventional thyroidectomy (range from 46.7 to 168.8 minutes). This observed difference in time required to carry out the same operation is quite striking. The heterogeneity may have been due to the size of the gland that was being resected, which was not clearly defined in all studies. In addition, all of the thyoidectomies in the Hallgrimsson study, which reported the longest mean operative time for conventional thyroidectomy, were carried out for Graves' thyrotoxicosis, wherein the vascularization of the thyroid gland can be very extensive. In contrast, the majority of thyroidectomies in the study reporting the fastest mean operative time excluded patients with Graves' disease or extensive goiters [12].

One must consider whether or not benign versus malignant thyroid pathology affected our results. All of the studies incorporated in the meta-analysis excluded patients requiring either a central or lateral compartment lymph node dissection; thus this could not have played a role in operative time or incidence of postoperative hypocalcemia. Of the 9 studies, 4 excluded malignant disease $(7,10,11,13), 3$ had no significant difference in the proportion of malignant cases between the HS and $\mathrm{CH}$ groups $(5,6,9), 1$ only included lowrisk T1N0M0 papillary thyroid cancers (12), and 1 did not clearly outline the pathologies. Given this, we do not feel that thyroid pathology is confounding our results for the primary or secondary outcomes.

Another consideration when interpreting the results of the current meta-analysis is that surgeons who conduct these trials may have significantly more experience with the HS than the average thyroid surgeon, and the timesaving effect of the HS might be exaggerated compared to what a lessfamiliar surgeon would experience when first adopting its use into their practice.

Future prospective, randomized trials of larger patient cohorts with more detailed and uniform definitions of postoperative complications, randomization procedures, intention-to-treat analyses, and blinding of outcome assessors are needed to draw more meaningful conclusions with regard to the influence of HS utilization on complications after total or subtotal thyroidectomy. In addition, costeffectiveness analyses to determine whether the costs saved from the reduced time spent in the operating theater outweigh the added cost of the HS scalpel would also be important. Several of the studies did report reduced overall cost associated with the HS $[7,8,10]$ while another reported no difference in overall costs when comparing the two techniques [5]. Other benefits seen with HS demonstrated in these studies included less operative bleeding [6, 8-12], fewer cases of postoperative hematoma formation [7], fewer ties used $[6,8,9,13]$, less drain utilization [13], less postoperative pain or analgesic requirements $[10,12]$, and smaller incisions [6]. The impact of a recently introduced, smaller handheld HS on thyroid surgery outcomes also warrants further study. Reports of the use of another vessel sealing technology, the Ligasure (Covidien, Boulder, Colorado), for thyroid surgery have emerged in the recent literature, and comparisons between this device and the HS would also be of interest. From the current study, we are able to definitively conclude that not only does the use of the HS significantly decrease operative time compared to $\mathrm{CH}$ techniques with ties, clips, and/or electrocautery but it is also safer in terms of reducing the incidence of transient postoperative hypocalcemia.

\section{Acknowledgments}

The authors would like to thank Dr. Andrea Petrucci and Mr. Antonio Canino for their help with translation of the French and Italian articles. In addition, they would like to thank 
Dr. Rob van Dam and Ms. Christie Jeon of the Harvard School of Public Health for their invaluable input. Dr. Wiseman is a Michael Smith Foundation for Health Research Scholar.

\section{References}

[1] L. Delbridge, "Total thyroidectomy: the evolution of surgical technique," ANZ Journal of Surgery, vol. 73, no. 9, pp. 761-768, 2003.

[2] S. Wiseman, P. Tomljanovich, and N. Rigual, "Thyroid lobectomy: operative anatomy, technique, and morbidity," Operative Techniques in Otolaryngology-Head and Neck Surgery, vol. 15, no. 3, pp. 210-219, 2004.

[3] G. D. Roye, J. Monchik, and J. F. Amaral, "Endoscopic adrenalectomy using ultrasonic cutting and coagulating," Surgical Technology International, vol. 9, pp. 129-138, 2000.

[4] W. S. Laycock, T. L. Trus, and J. G. Hunter, "New technology for the division of short gastric vessels during laparoscopic Nissen fundoplication. A prospective randomized trial," Surgical Endoscopy, vol. 10, no. 1, pp. 71-73, 1996.

[5] C. P. Lombardi, M. Rafaelli, A. Cicchetti, et al., "The use of "harmonic scalpel" versus "knot tying" for conventional "open" thyroidectomy: results of a prospective randomized study," Langenbeck's Archives of Surgery, vol. 393, no. 5, pp. 627-631, 2008.

[6] M. Kilic, M. Keskek, T. Ertan, et al., "A prospective randomized trial comparing the harmonic scalpel with conventional knot tying in thyroidectomy," Advances in Therapy, vol. 24, no. 3, pp. 632-638, 2007.

[7] J. Ortega, C. Sala, B. Flor, et al., "Efficacy and cost-effectiveness of the UltraCision harmonic scalpel in thyroid surgery: an analysis of 200 cases in a randomized trial," Journal of Laparoendoscopic and Advanced Surgical Techniques A, vol. 14, no. 1, pp. 9-12, 2004.

[8] M. Frazzetta, G. Furgiuele, D. Raimondo, et al., "Ultrasonic dissector for total thyroidectomy: results of prospective randomized study," Giornale di Chirurgia, vol. 26, no. 8-9, pp. 295-301, 2005.

[9] C. Cordon, R. Fajardo, J. Ramirez, et al., "A randomized, prospective, parallel group study comparing the harmonic scalpel to electrocautery in thyroidectomy," Surgery, vol. 137, no. 3, pp. 337-341, 2005.

[10] T. Defechereux, F. Rinken, S. Maweja, et al., "Evaluation of the ultrasonic dissector in thyroid surgery. A prospective randomised study," Acta Chirurgica Belgica, vol. 103, no. 3, pp. 274-277, 2003.

[11] P. Hallgrimsson, L. Loven, J. Westerdahl, et al., "Use of the harmonic scalpel versus conventional haemostatic techniques in patients with Grave disease undergoing total thyroidectomy: a prospective randomised controlled trial," Langenbeck's Archives of Surgery, vol. 393, no. 5, pp. 675-680, 2008.

[12] P. Miccoli, P. Berti, G. Dionigi, et al., "Randomized controlled trial of harmonic scalpel use during thyroidectomy," Archives of Otolaryngology - Head and Neck Surgery, vol. 132, no. 10, pp. 1069-1073, 2006.

[13] O. Yildirim, T. Umit, M. Ebru, et al., "Ultrasonic harmonic scalpel in total thyroidectomies," Advances in Therapy, vol. 25, no. 3, pp. 260-265, 2008.

[14] M. Marchesi, M. Biffoni, R. Cresti, et al., "Ultrasonic scalpel in thyroid surgery," Chiropractic in Italy, vol. 55, no. 2, pp. 299 308, 2003.
[15] P. E. Voutilainen and C. H. Haglund, "Ultrasonically activated shears in thyroidectomies: a randomized trial," Annals of Surgery, vol. 231, no. 3, pp. 322-328, 2000.

[16] R. DerSimonian and N. Laird, "Meta-analysis in clinical trials," Controlled Clinical Trials, vol. 7, no. 3, pp. 177-188, 1986.

[17] C. B. Begg and M. Mazumdar, "Operating characteristics of a rank correlation test for publication bias," Biometrics, vol. 50, no. 4, pp. 1088-1101, 1994.

[18] M. Egger, G. Davey Smith, M. Schneider, et al., "Bias in metaanalysis detected by a simple, graphical test," British Medical Journal, vol. 315, no. 7109, pp. 629-634, 1997.

[19] A. Salami, M. Bavazzano, R. Mora, et al., "Harmonic scalpel in pharyngolaryngectomy with radical neck dissection," Journal of Otolaryngology-Head and Neck Surgery, vol. 37, no. 5, pp. 633-637, 2008.

[20] S. S. Bessa, T. A. Al-Fayoumi, K. M. Katri, et al., "Clipless laparoscopic cholecystectomy by ultrasonic dissection," Journal of Laparoendoscopic and Advanced Surgical Techniques A, vol. 18, no. 4, pp. 593-598, 2008.

[21] N. Druzijanic, Z. Perko, D. Kraljevic, et al., "Harmonic scalpel in transanal microsurgery," Hepatogastroenterology, vol. 55, no. 82-83, pp. 356-358, 2008.

[22] L. Shemen, "Thyroidectomy using the harmonic scalpel: analysis of 105 consecutive cases," Otolaryngology-Head and Neck Surgery, vol. 127, no. 4, pp. 284-288, 2002.

[23] A. E. Siperstein, E. Berber, and E. Morkoyun, "The use of the harmonic scalpel vs conventional knot tying for vessel ligation in thyroid surgery," Archives of Surgery, vol. 137, no. 2, pp. 137$142,2002$. 


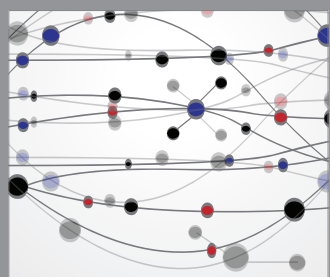

The Scientific World Journal
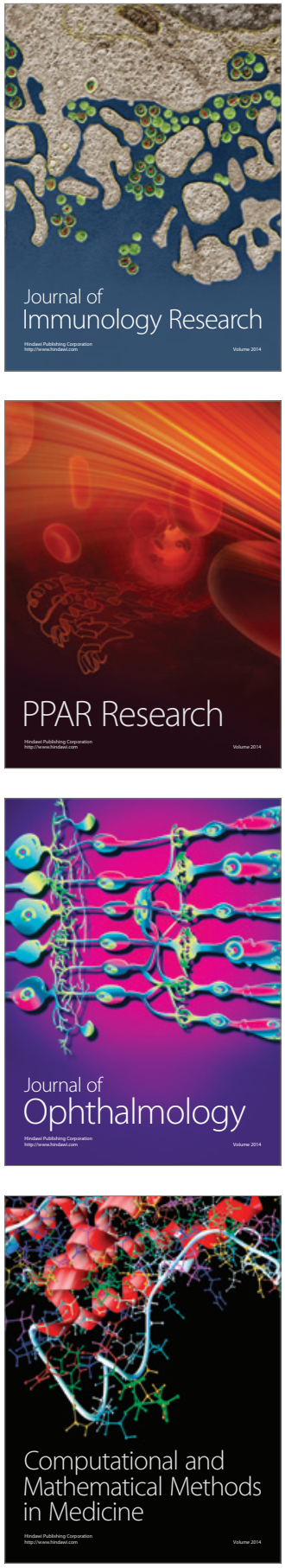

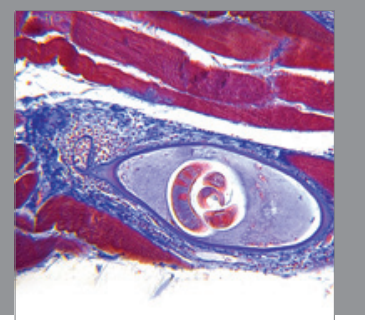

Gastroenterology

Research and Practice
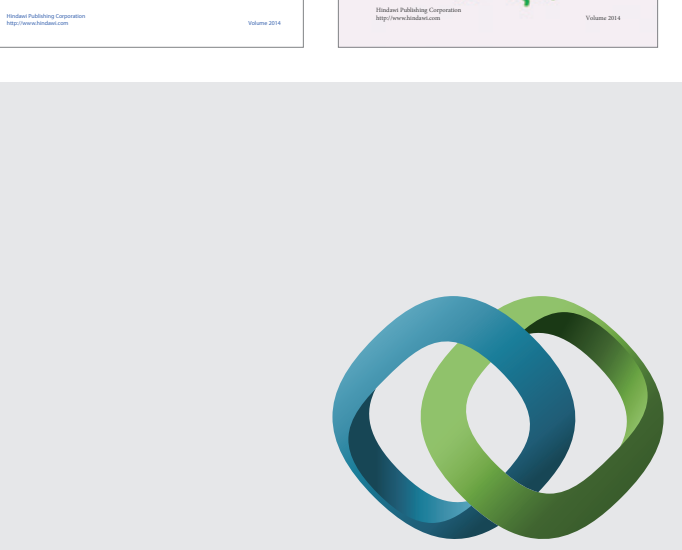

\section{Hindawi}

Submit your manuscripts at

http://www.hindawi.com
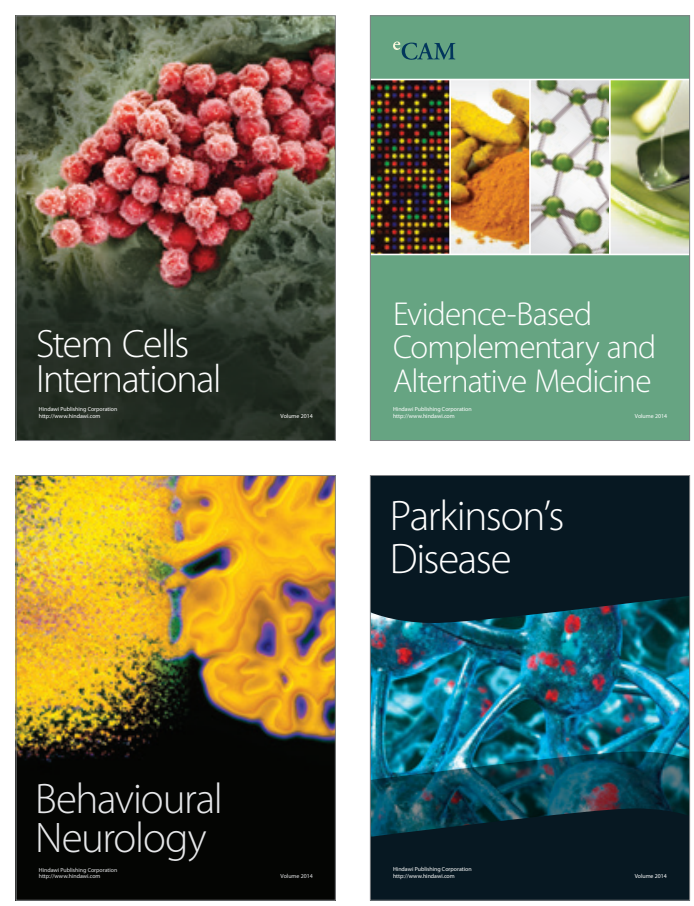

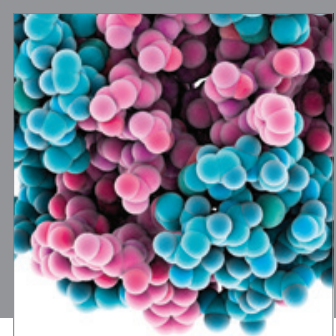

Journal of
Diabetes Research

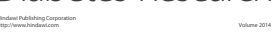

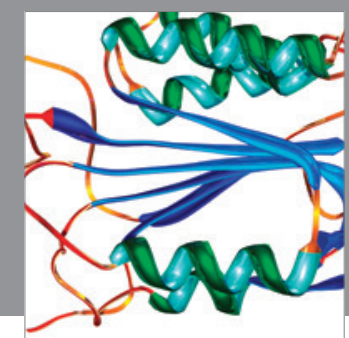

Disease Markers
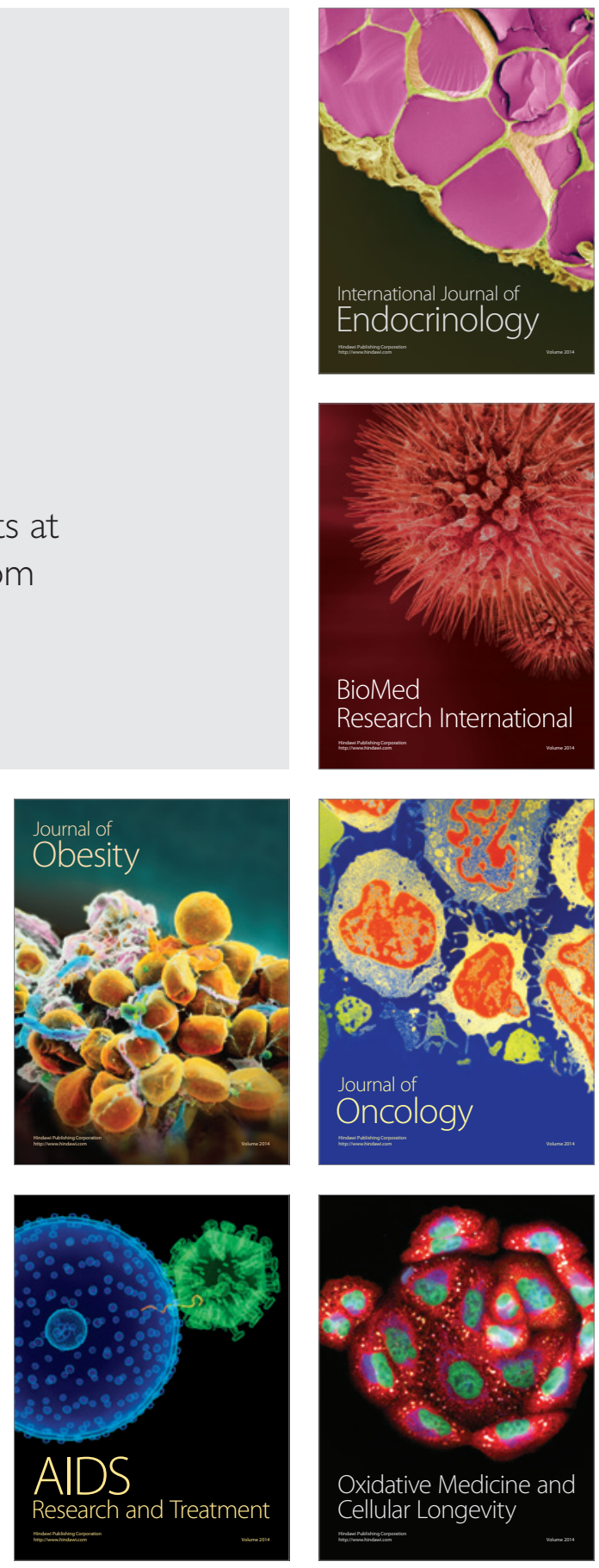